\title{
Effects of Irradiance and Stimulus Duration on Early Gene Expression (Fos) in the Suprachiasmatic Nucleus: Temporal Summation and Reciprocity
}

\author{
O. Dkhissi-Benyahya, ${ }^{1}$ B. Sicard, ${ }^{2}$ and H. M. Cooper ${ }^{1}$ \\ 1/nstitut National de la Santé et de la Recherche Médicale Unité 371, Cerveau et Vision, 69675 Bron, France, and \\ 2Laboratoire de Mammalogie, Institut de Recherche pour le Developpement, BP 2528, Bamako, Mali
}

The daily behavioral, physiological, and hormonal rhythms in mammals are regulated by an endogenous circadian clock located in the suprachiasmatic nucleus (SCN) and are synchronized by the natural $24 \mathrm{hr}$ light/dark cycle. We studied the response properties (threshold, saturation, and linearity) of the photic system to irradiance by assaying light induction of Fos, the protein product of the immediate early gene $c$-fos. Fos expression was quantified by image analysis in the SCN and in the retina. Fos expression in the SCN and retina are unrelated because the response differs in terms of threshold, saturation, and range. In the SCN, Fos expression increases proportionately to increases in both irradiance and duration of light exposure.
The photic system shows a linear temporal integration of photons for durations ranging from $3 \mathrm{sec}$ to $47.5 \mathrm{~min}$. The principal result of this study shows that in the SCN, Fos expression is directly proportional to the total number of photons rather than to irradiance or duration alone (reciprocity), and that integration occurs over a range of $5 \mathrm{log}$ units of photon number. This report provides the first demonstration that the mechanism of photon integration by the circadian system is expressed at a cellular level in the SCN.

Key words: suprachiasmatic nucleus; immediate early genes; circadian system; photoreception; retina; endogenous rhythms
The characterization of the mechanisms by which light influences the phase of the circadian oscillator is one of the major problems that must be addressed to understand circadian physiology (Pittendrigh, 1981). Studies in rodents suggest that the coupling of light information to intracellular signaling pathways in the suprachiasmatic nucleus (SCN) involves the proto-oncogene $c$-fos, an immediate early gene, and its product, the phosphoprotein Fos. (1) The photic induction of $c$-fos is restricted mainly to the ventrolateral part of the SCN, in the region of retinal terminals (Aronin et al., 1990; Earnest et al., 1990; Rusak et al., 1990). (2) Pharmacological agents that block behavioral phase shifts also block the photic stimulation of $c$-fos in the SCN of hamster (Vindlacheruvu et al., 1992; Colwell et al., 1993a; Rea et al., 1993). (3) Light-induced phase shifts of rat circadian locomotor activity are prevented by intracerebroventricular injections of antisense oligodeoxynucleotides against both $c$-fos and JunB (Wollnik et al., 1995). (4) Lightinduced Fos expression is characterized by a phase dependence similar to that for light-induced phase shifts of locomotor activity (Aronin et al., 1990; Kornhauser et al., 1990, 1992; Rusak et al., 1990, 1992; Colwell and Foster, 1992; Schwartz et al., 1994; Cooper et al., 1998).

In both animals and humans the amplitude of circadian responses such as a phase shift of locomotor activity and light suppression of plasma melatonin are correlated with the level of irradiance (Brainard et al., 1988; Kornhauser et al., 1990; Foster et al., 1991; Nelson and Takahashi, 1991a,b; Bauer, 1992; Boivin et al., 1996; Yoshimura and Ebihara, 1996). In addition, the degree of $c$-fos mRNA or Fos protein induction is correlated with irradiance and the amplitude of a phase shift, although only a limited range of irradiance levels has been studied (Kornhauser et al., 1990; Shimomura et al., 1998; Lupi et al., 1999).

\footnotetext{
Received July 13, 2000; accepted July 20, 2000.

This research was funded by grants from Human Frontier (RG95/68), Biomed2 (BMH4-CT972327), and NATO (950334). We thank Christel Merrouche and Naura Chounlamountri for technical assistance.

Correspondence should be addressed to Dr. H. M. Cooper, Institut National de la Santé et de la Recherche Médicale, Unité 371, Cerveau et Vision, 18 Avenue du Doyen Lépine, 69675 Bron, France. E-mail cooper@lyon151.inserm.fr.

Copyright (C) 2000 Society for Neuroscience $0270-6474 / 00 / 207790-\bullet \$ 15.00 / 0$
}

In contrast, relatively few studies have attempted to quantify responses of the circadian system to stimulus duration. A single short pulse of light $(<1 \mathrm{sec})$ fails to induce a phase shift of locomotor activity, which generally requires longer durations of light exposure. Studies of temporal response properties have suggested that the circadian system may function as a "photon counter" by integrating the number of photons in the light stimulus over extended periods of time (Takahashi et al., 1984; Nelson and Takahashi, 1991a, 1999). For stimulus durations in the range of 300 sec, the amplitude of a behavioral phase shift is directly dependent on the total photon number in the light stimulus. However, the efficiency of the system is decreased for short ( $<30 \mathrm{sec})$ and long (1 hr) duration pulses.

The stimulus-response relationships for irradiance and duration in relation to $c$-fos induction in the SCN have not been clearly defined. Because both behavioral phase shifts and $c$-fos induction are dependent on irradiance, the question arises as to whether temporal integration of photons, and reciprocity for irradiance and duration, hold for light-induced $c$-fos expression. The issue of whether reciprocity for stimulus irradiance-duration is expressed at a cellular level is important, because the mechanism and site of photon integration in the circadian system are unknown.

\section{MATERIALS AND METHODS}

Animals and photic stimulation. Gerbils (Taterillus petteri) were captured in the field in the region of Bamako, Mali, 3-4 weeks before testing and maintained in the laboratory in individual cages on a diet of rodent pellets and apples. This species was used as a model for these investigations because substantial data are available on Fos induction (Cooper et al., 1998), and this rodent is of considerable economic importance (Sicard et al., 1999). All animals were kept for at least $10 \mathrm{~d}$ under a $12 \mathrm{hr}$ light/dark cycle using fluorescent lights $(\sim 300$ lux $)$ before the experiments. On the day of testing, the light/dark cycle was discontinued, and the gerbils were maintained in constant darkness. Thus, the time at which light onset would normally have occurred (ZT0) was used as the beginning of the subjective day (CT0) (Schwartz et al., 1994). Animals were stimulated with a light pulse at CT16, because this time corresponded to the period of the circadian cycle of maximum amplitude of Fos expression in the gerbil (Cooper et al., 1998)

In the series tested for irradiance, animals $(n=41)$ were exposed to a $15 \mathrm{~min}$ monochromatic pulse of light $(\lambda=500 \mathrm{~nm})$ at different irradiance levels. The wavelength and level of irradiance were varied using interference and neutral density filters (see below for details of light stimulation 
and measurement). Light irradiance extended over $7 \log$ units from $3.8 \times$ $10^{-5}$ to $3.8 \times 10^{2} \mu \mathrm{W} / \mathrm{cm}^{2}$. After the light pulse, animals were returned to complete darkness and perfused $60 \mathrm{~min}$ after the beginning of the light pulse.

In the series tested for stimulus duration, animals $(n=45)$ were exposed to a $500 \mathrm{~nm}$ monochromatic pulse of light at a constant irradiance $3.8 \times$ $\left.10^{-1} \mu \mathrm{W} / \mathrm{cm}^{2}\right)$ but of variable duration in one-half log unit steps $(3,9,15$, $28,90,285,900,2850 \mathrm{sec})$. Subsequently, animals were returned to complete darkness until perfusion, which was performed $60 \mathrm{~min}$ after the beginning of the light pulse. For each time point of each series, three to four animals were examined for the presence of Fos protein in the SCN and in the retina using immunohistochemistry. All handling and transfer of the animals were performed in light-tight rooms in complete darkness. Control animals (six for each series) were handled identically but were no exposed to light. Data for the analysis of Fos induction in the SCN and retina were obtained from the same individual animals of each of the above series. All treatment of animals was in strict accordance with current international regulations on animal care, housing, and experimentation.

The stimulus apparatus used a tungsten-halogen light source (24 V-150 W) equipped with condenser and collimating lenses and an infrared absorbing filter. Monochromatic light was produced with a Schott interference filter, and irradiance varied with neutral density filters (Eastman Kodak, Rochester, NY). Peak irradiance and spectral output $(\lambda=500 \mathrm{~nm}$, half band width $9.34 \mathrm{~nm}$ ) were measured in the stimulation chamber using an International Light model IL 700 photometer and a spectrophotometer (Ocean Optics S2000). Irradiance of the stimulus was measured in microwatts per centimeters squared and converted to photons per centimeters squared per second based on the energy per photon for $\lambda=500 \mathrm{~nm}$. All results are presented as both photon flux and microwatts per centimeters squared. The cylindrical chamber (diameter $=14 \mathrm{~cm}$, height $=7 \mathrm{~cm}$ ) was coated with reflective neutral white enamel. Light was projected into the stimulation chamber through an opal diff user ( $3 \mathrm{~mm}$ thick). The homogeneity of transmission over the surface of the diffuser and the spectral reflectance of the chamber were verified with the spectrophotometer. The entire stimulator (light source and chamber) was enclosed in a light-tight box. Onset and duration of the light pulse were controlled with electronic timers.

Fos immunohistochemistry. Animals were rapidly anesthetized in complete darkness with halothane followed by an injection of nembutal (100 $\mathrm{mg} / \mathrm{kg}$, i.p.) and then perfused intracardially (in dim light with the head covered) with warm $\left(37^{\circ} \mathrm{C}\right.$ ) heparinized saline followed by $300 \mathrm{ml}$ of Zamboni's fixative at $4^{\circ} \mathrm{C}$. The anesthesia always began $60 \mathrm{~min}$ after the beginning of the light stimulation, and perfusion was initiated within 2-3 min thereafter. Brains and eyes were post-fixed overnight in the same fixative at $4^{\circ} \mathrm{C}$, removed from the skull, and subsequently stored in phosphate buffer with $0.1 \%$ sodium azide. Before sectioning, brains were cryoprotected in $30 \%$ sucrose phosphate buffer for $24 \mathrm{hr}$. Serial coronal sections were made at $40 \mu \mathrm{m}$ on a freezing microtome. The retinas were removed from the eye and dissected free from the pigment epithelium, and the vitreous was cleaned away. Free floating retinas were processed identically to brain sections for Fos immunohistochemistry. All sections of series 1 and 2 were processed simultaneously to obtain identical levels of tissue staining for data analysis. Endogenous peroxidase was first suppressed using a solution of $50 \%$ ethanol in saline with $0.03 \% \mathrm{H}_{2} \mathrm{O}_{2}$. Free-floating sections were rinsed briefly in PBS (0.01 M, pH 7.2) containing $0.3 \%$ Triton and $0.1 \%$ sodium azide and blocked with $1.5 \%$ norma goat serum. Sections were incubated in the anti-Fos primary antibody (Ab-5 rabbit antiserum, Oncogene Research Products, Calbiochem, La Jolla, CA; dilution 1:10,000) for $3 \mathrm{~d}$ at $4^{\circ} \mathrm{C}$. Immunoreactivity was visualized using a Vectastain ABC Elite kit (PK-6100, Vector Laboratories, Burlingame, CA), followed by incubation in $0.2 \% 3,3^{\prime}$-diaminobenzidine with $0.5 \%$ ammonium nickel sulfate and $0.003 \% \mathrm{H}_{2} \mathrm{O}_{2}$ in Tris buffer $(0.05$ $\mathrm{M}, \mathrm{pH}$ 7.6). Controls sections were made by preincubation of the primary antibody with Fos peptide (Oncogene Research, PP10), by omitting the primary antiserum or by replacement with normal serum at the same concentration as the antibody. No labeling was observed in control sections.

Quantitative analysis of Fos induction. Quantitative levels of Fos-like immunopositive product in the SCN were determined using computerassisted image analysis (Biocom, Les Ulis, France). Optical density of label was measured bilaterally from digitized images of the SCN. This method is based on quantification of the total optical density of Fos labeling, defined as the integral sum of the surface area of all pixels in the SCN multiplied by their corresponding optical density value. The optical density was obtained by first subtracting the background density value determined from an adjacent area of hypothalamus that did not contain Fos-positive cells. The background for each animal was systematically measured and in general did not vary more than $\pm 3 \%$. A threshold value was determined for detection of label significantly above the background level. The identity of the individual animals was unknown to the observer during the analysis, and every section of the SCN of each series was analyzed.

For valid comparative measures using image analysis, reduction of variations in the light source and the constancy of the histological methods are imperative. The intensity of the microscope light source $(12 \mathrm{~V}$ tungsten-halogen lamp) was held constant using a $220 \mathrm{~V}$ stabilizer (500 VA) and a stabilized $220 \mathrm{~V}-12 \mathrm{~V}$ transformer. A fiber optic coupler between the light source and microscope was used to finely adjust light levels and provide uniform illumination. To assure uniformity over the digitized field, differences in density caused by optical distortion were corrected by subtracting the bright-field background image (blank slide) from the digitized image. Room temperature was also held relatively constant, because the output response of digital cameras can vary with a difference of a few degrees. The irradiance level of the microscope field was verified between measures of each animal. Identical histological treatment is also important because previous tests have also shown that direct quantitative comparisons between different animals require that all sections of the series be treated simultaneously in the same reaction trays.

Ganglion cell density counts. The number of Fos-positive nuclei in the retinal ganglion cell layer was quantified in the following way. An estimation of the total number of Fos-positive nuclei was made by moving a $0.15 \times 0.15-\mathrm{mm}$-square graticule sample surface over the whole retina using a $25 \times$ objective. Five samples were collected within each of the four main retinal quadrants; upper, lower, nasal, and temporal were counted. The number of cells was expressed as the mean number of cells per micrometers squared of retina.

Choice of the analysis methods. To analyze Fos induction in the SCN, we used an image analysis rather than the usual cell counting method for several reasons. (1) The analysis of the optical density is an objective measure because constant conditions and a threshold value are applied for the entire analysis. Counting cells is more subjective because a precise threshold value cannot be quantitatively fixed and can vary according to the observer, illumination, and other factors. (2) Quantitative immunohistochemical studies of several neurotransmitters have shown that optical density is proportional to the quantity of immunolabel in tissue sections (Nabors et al., 1988; Jojich and Pourcho, 1996). Preliminary results in our laboratory also show that measures of optical density are linearly proportional to Fos peptide concentration (C. Rieux, unpublished observations) Cell counts do not take into account the difference in the quantity of label between cells, thus giving equal weight to lightly or densely stained cells. (3) The image analysis method of optical density allows determination of the total optical density in all sections of the SCN (and from a large sample of animals), whereas direct cell counts are usually performed only in a small number of sections, which may thus introduce bias. (4) Counting cells in thick sections without use of stereological methods is inaccurate.

For the retina, we counted cells localized in the ganglion cell layer rather than measuring the optical density. Because of the greater thickness of the retina (superior to $200 \mu \mathrm{m}$ ), as well as regional variations in thickness from center to periphery (and thus variations in background), plus scattered deposits of pigment epithelium, it is difficult to apply a constant threshold value between retinas or within the same retina. Stereological methods for counting cells in the ganglion cell layer of retina are not necessary because neurons can be visualized throughout the entire thickness.

Statistical analyses and terminology. To compare the relationship between stimulus irradiance and duration in the retina and the SCN, the data were fit with a four-parameter logistic equation using a modified form of the Naka-Rushton equation (Naka and Rushton, 1966). For this function:

$$
R=\frac{R_{\min }-R_{\max }}{1+(I / \sigma)^{\mathrm{p}}}+R_{\max },
$$

where $I$ is the value of the light stimulus (irradiance, duration, or the total number of photons) and the response, $R$, is the total optical density of Fos labeling. $R_{\max }$ is the maximum response to the stimulus parameter, $I$, and $R_{\min }$ is the response when $I=0$. The stimulus value required to induce a response of one-half the maximum response is $\sigma . p$ is the slope of the function.

Statistical analyses were performed using a one-way ANOVA followed, when significant $(p<0.05)$, by Student's $t$ test for a comparisons of two groups. Results in the graphs (see Figs. 1-3) are shown as SEM.

Several terms are generally used for the description of response properties. "Sensitivity" usually refers to the reciprocal of the stimulus required to produce a response of a criterion value. In psychophysics, the magnitude of the stimulus required to elicit a one-half maximum response is usually used, because the response measured represents a probability of observing a given effect. This definition of sensitivity is independent of the maximum response but is dependent on the range (interval between threshold and saturation) and slope of the response in given stimulus conditions. "Threshold" refers to the stimulus quantity required to induce a response of a criterion value, or of statistical significance compared with control values. "Saturation" refers to the stimulus quantity beyond which response amplitude shows no significant increase.

\section{RESULTS}

\section{Fos induction in the SCN in response to stimulus irradiance and duration}

Previous studies have shown that Fos induction in the SCN is related to the level of irradiance (Kornhauser et al., 1990; Zhang et al., 1996; Lupi et al., 1999). In the SCN, the lowest irradiance stimuli delivered in our study $\left(0.95 \times 10^{8}\right.$ photons per $\mathrm{cm}^{2} / \mathrm{sec}$, corresponding to $3.8 \times 10^{-5} \mu \mathrm{W} / \mathrm{cm}^{2}$ ) induces significant Fos 

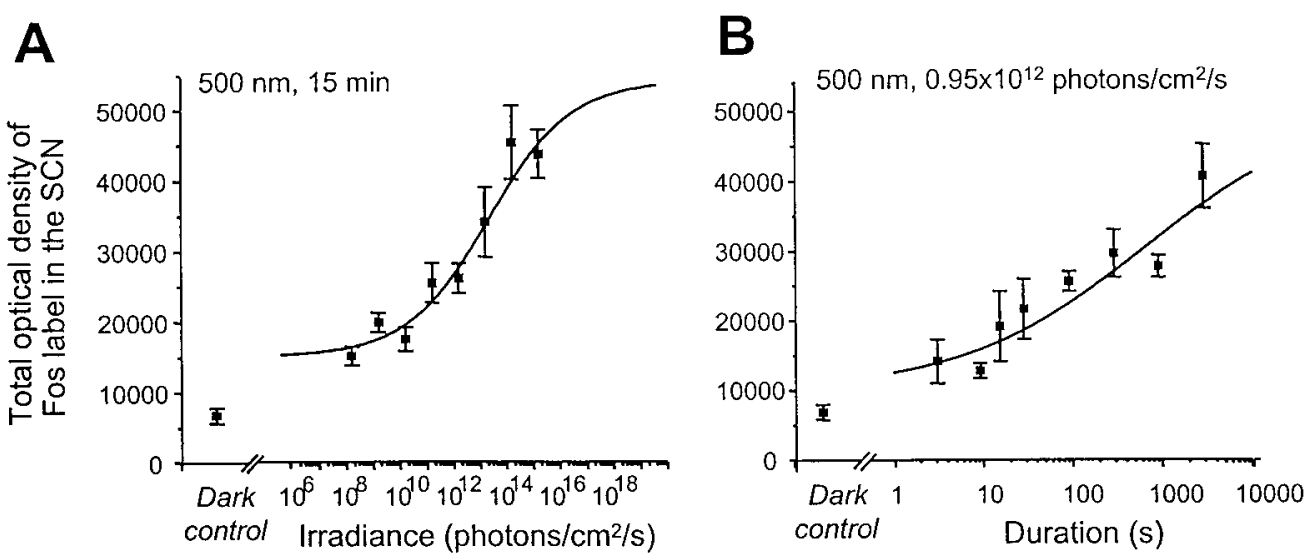

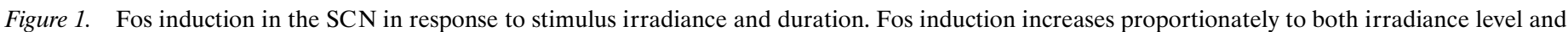

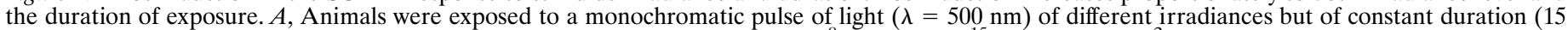

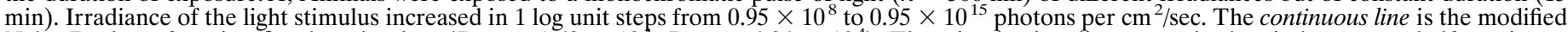

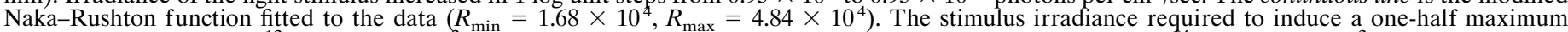

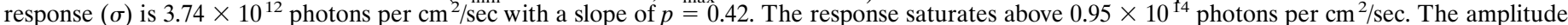

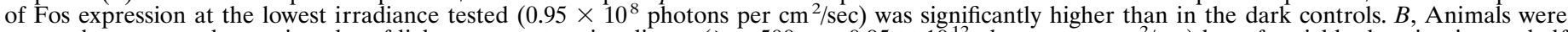

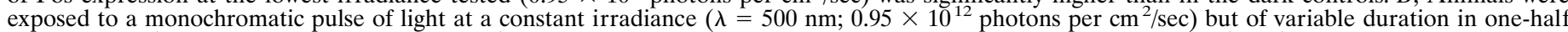

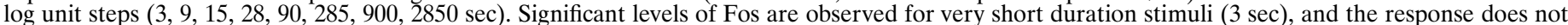

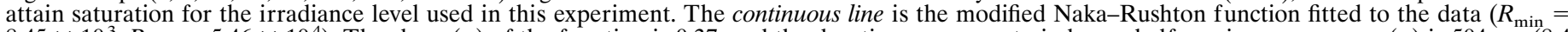

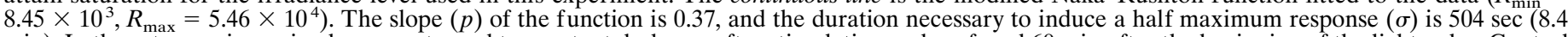

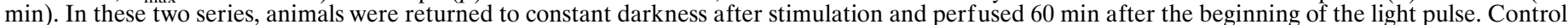
animals were handled identically but were not exposed to light. Error bars represent SEM ( $n=4$ for each point).

expression $(p<0.05)$ at CT16 compared with dark control animals (Figs. $1 A, 4 A$ ). Fos expression remains at reduced but constant levels from $0.95 \times 10^{8}$ to $0.95 \times 10^{10}$ photons per cm ${ }^{2} / \mathrm{sec}(3.8 \times$ $10^{-5}$ to $\left.3.8 \times 10^{-3} \mu \mathrm{W} / \mathrm{cm}^{2}\right)$. Subsequently, the density of Fos induction in the SCN increases proportionately with increasing irradiance levels from $0.95 \times 10^{10}$ to $0.95 \times 10^{14}$ photons per $\mathrm{cm}^{2} / \mathrm{sec}\left(3.8 \times 10^{-3}\right.$ to $\left.3.8 \times 10^{1} \mu \mathrm{W} / \mathrm{cm}^{2}\right)$ (Figs. $\left.1 A, 4\right)$. At this level of irradiance $\left(0.95 \times 10^{14}\right.$ photons per $\left.\mathrm{cm}^{2} / \mathrm{sec}\right)$, the response reaches saturation levels, with no further increase observed at the highest irradiance used $\left(0.95 \times 10^{15}\right.$ photons per $\mathrm{cm}^{2} / \mathrm{sec}, 3.8 \times$ $10^{2} \mu \mathrm{W} / \mathrm{cm}^{2}$ ) (Figs. $1 A, 4 G$ ). The irradiance threshold for induction of Fos in the SCN in the hamster was originally described to be in the range of $10^{11}$ photons per $\mathrm{cm}^{2} / \mathrm{sec}$ (Kornhauser et al., 1990). A subsequent study from the same group (Zhang et al., 1996) observed a lower threshold of $7.5 \times 10^{9}$ photons per $\mathrm{cm}^{2} / \mathrm{sec}$ $\left(2.9 \times 10^{-3} \mu \mathrm{W} / \mathrm{cm}^{2}\right)$, similar to that required for a behavioral phase shift (Nelson and Takahashi, 1991a; Zhang et al., 1996). In our study, we observed a significant induction of Fos at an irradiance level almost $2 \log$ units less. This value is nevertheless several $\log$ units above scotopic responses in rodents or humans (Dodt and Echte, 1961; Le Grand, 1968; Emerson, 1980). The differences in the absolute values of the thresholds may simply be related to the fact that few studies have examined the effects of very low irradiance levels. Indeed, there is some suggestion that at very low light levels $\left(10^{-4} \mu \mathrm{W} / \mathrm{cm}^{2}\right)$, a behavioral effect is not totally absent (Foster et al., 1991, their Fig. 3; Nelson and Takahashi, 1991a, their Fig. 3B). The irradiance required for saturation differs, because Fos expression saturates above $10^{14}$ photons per $\mathrm{cm}^{2} / \mathrm{sec}$, whereas the phase shift response saturates at $\sim 10^{13}$ photons per $\mathrm{cm}^{2} / \mathrm{sec}$.

When the density of light-induced Fos expression is plotted as a function of $\log _{10}$-stimulus irradiance, the data can be fitted by a four-parameter curve (Naka and Rushton, 1966). The slope of the function was 0.42 , and the irradiance necessary to induce a half maximum response was $3.74 \times 10^{12}$ photons per $\mathrm{cm}^{2} / \mathrm{sec}(1.49$ $\left.\mu \mathrm{W} / \mathrm{cm}^{2}\right)$.

To determine the photic response to different stimulus durations, the degree of Fos induction was measured after 3, 9, 15, 28.5, 90, 285,900 , and $2850 \mathrm{sec}$ of light stimulation at a constant irradiance level $\left(0.95 \times 10^{12}\right.$ photons per $\left.\mathrm{cm}^{2} / \mathrm{sec}, 3.8 \times 10^{-1} \mu \mathrm{W} / \mathrm{cm}^{2}\right)$, which is essentially equal to the one-half saturation value determined above. We demonstrate for the first time that Fos levels in the $\mathrm{SCN}$ increase proportionately with an increase in the duration of the light stimulus. In the gerbil, a $3 \mathrm{sec}$ light duration is sufficient to induce significant levels of Fos $(p<0.05)$ in the SCN. Fos levels then increase proportionately with increasing stimulus duration (Fig. $1 B$ ) up to $47.5 \mathrm{~min}(2850 \mathrm{sec})$. The magnitude of the response of light-induced Fos expression was fit to log stimulus duration by a four-parameter curve. At the longest stimulus duration in our study (47.5 min), the Fos response did not appear to saturate (see Discussion), suggesting that the duration used in this study is not limiting. The slope of the function is 0.37 , which is similar to that of the irradiance response curve. The duration necessary to induce a half-maximum response was $504 \mathrm{sec}(\sim 10 \mathrm{~min})$.

\section{Fos induction in the retina in response to stimulus irradiance and duration}

The responses of Fos induction to variations in irradiance and duration of the light stimulus have never been studied in the retina, although several investigations have reported Fos protein and $c$-fos mRNA induction by light in retinal neurons (Earnest et al., 1990; Sagar and Sharp, 1990; Chambille et al., 1993; Nir and Agarwal, 1993; Yoshida et al., 1993). In the retina, Fos induction in relation to light stimulation shows a distinctly different pattern from that in the SCN in terms of threshold, slope, and saturation levels. A significantly higher level of irradiance $\left(0.95 \times 10^{12}\right.$ photons per $\left.\mathrm{cm}^{2} / \mathrm{sec}, 3.8 \times 10^{-1} \mu \mathrm{W} / \mathrm{cm}^{2}\right)$ is required to induce any observable Fos expression in the ganglion cell layer (Figs. $2 A, 4 F-H)$ ). At this irradiance $\sim 380$ cells $/ \mathrm{mm}^{2}$ are present. These Fos-immunoreactive profiles were presumed to correspond to displaced amacrine and ganglion cells. Below this level, and in the dark control animals, fewer than 12 cells $/ \mathrm{mm}^{2}$ are seen in the ganglion cell layer (Fig. $4 B, D)$. In contrast to the $\mathrm{SCN}$, Fos induction saturates rapidly at $0.95 \times 10^{13}$ photons per $\mathrm{cm}^{2} / \mathrm{sec}\left(3.8 \times 10^{0} \mu \mathrm{W} / \mathrm{cm}^{2}\right)($ Fig. $2 A)$. By fitting the data for the response for Fos-positive ganglion cells to irradiance with a four-parameter curve, we find that the slope of the curve is relatively steep (1.41), and the irradiance necessary to induce a half-maximum response was $3.0 \times 10^{11}$ photons per $\mathrm{cm}^{2} / \mathrm{sec}\left(0.12 \mu \mathrm{W} / \mathrm{cm}^{2}\right)$.

The threshold for Fos induction in the retina to photic stimuli of different durations also requires higher levels of irradiance compared with the SCN. A significant level of Fos induction is only observed after $900 \mathrm{sec}(15 \mathrm{~min})$ and $2850 \mathrm{sec}(47.5 \mathrm{~min})$ of continuous light stimulation (Fig. $2 B$ ). The slope of the curve was 0.9 , resulting in a narrow stimulus-response range $(\mathrm{SCN}=5 \mathrm{log}$ units, 

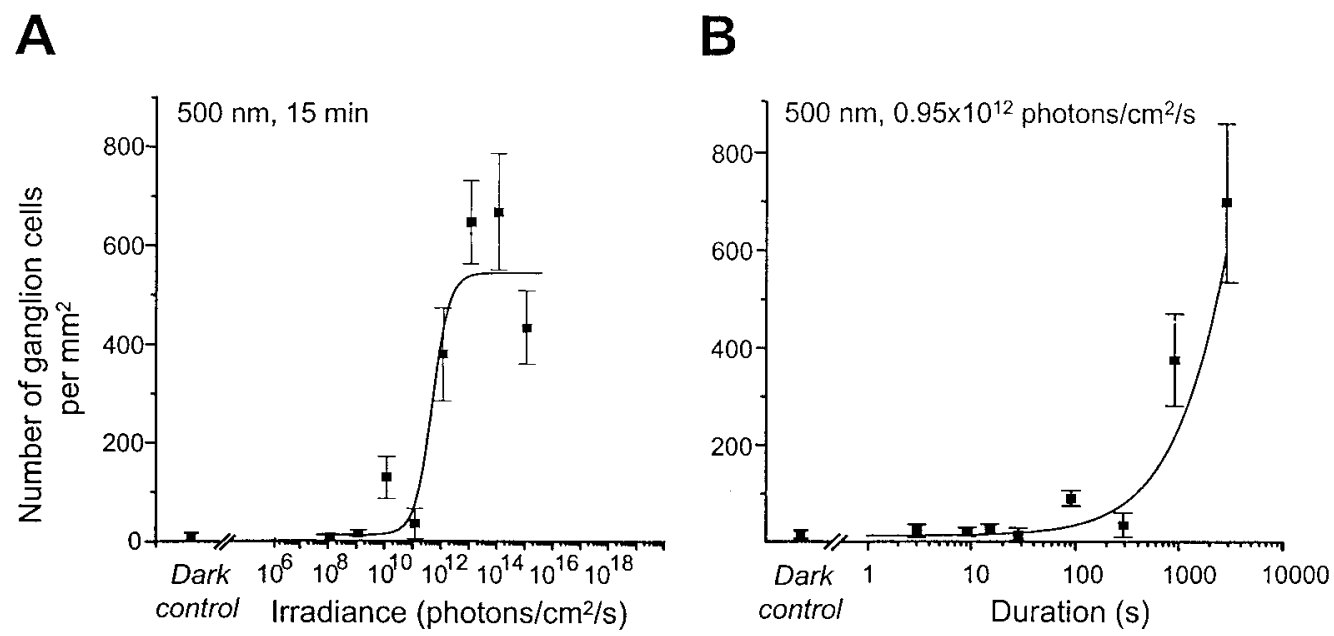

Figure 2. Fos induction in the ganglion cell layer of the retina in response to stimulus irradiance $(A)$ and duration $(B)$. Experimental conditions and stimulus irradiance and duration were the same as in Figure 1. The data points represent retinas from the same individuals used in Figure 1. The mean number of Fos-immunopositive nuclei (cells per millimeters squared) were counted in the ganglion cell layer over the surface of whole retinal flatmounts. $A$, In the retina, the number of Fos-immunopositive cells increases sharply at a relatively high level of irradiance $\left(0.95 \times 10^{12}\right.$ photons per $\left.\mathrm{cm}^{2} / \mathrm{sec}\right)$. We fitted the density of Fos-positive ganglion cells using a four-parameter curve. The continuous line is the modified Naka-Rushton function fitted to the data $\left(R_{\min }=10.10, R_{\max }=543.2\right)$. The slope $(p)$ of the curve is relatively steep (1.41), and the irradiance necessary to induce a half maximum response is $3.0 \times$ $10^{\mathrm{TI}}$ photons per cm$/ \mathrm{sec} B$, For the response to different stimulus durations, relatively long durations are required to induce Fos. The continuous line is the modified Naka-Rushton function fitted to the data $\left(R_{\min }=16.67, R_{\max }=701.5, p=0.9\right.$, and $\left.\sigma=450 \mathrm{sec}\right)$.

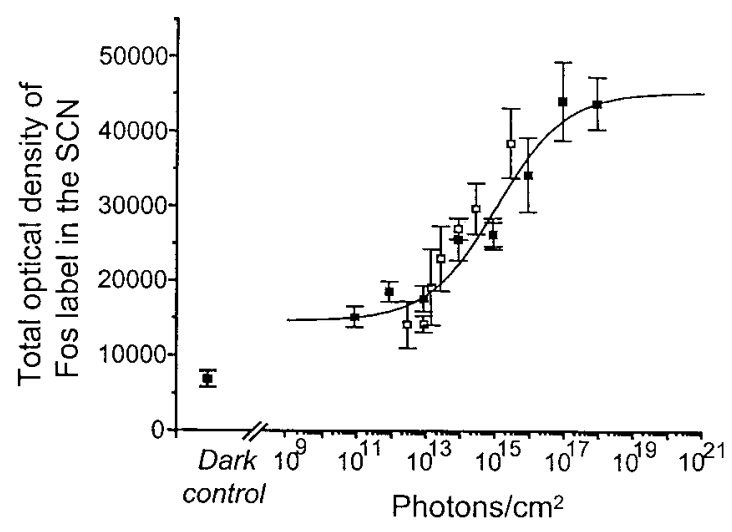

Figure 3. Induction of Fos in the SCN in response to the total number of photons. The data from Figure 1 for the SCN were plotted as a function of the total number of photons in the light stimulus. This figure shows that light-evoked Fos expression in the SCN is proportional to the total number of photons in the range of $10^{12}-10^{17}$ photons $/ \mathrm{cm}^{2}$, rather than irradiance or duration alone ( $=$ data points from irradiance response curve, Fig. $1 A$; $\square=$ data points from duration response curve, Fig. $1 B$ ). The continuous line is the modified Naka-Rushton function fitted to the data $\left(R_{\min }=\right.$ $\left.1.47 \times 10^{4}, R_{\max }=4.53 \times 10^{4}\right)$. The number of photons required to induce a one-half maximum response $(\sigma)$ is $1.088 \times 10^{15}$ photons $/ \mathrm{cm}^{2}$, and the slope $(p)$ is $=0.44$

retina $=2 \log$ units). In our experimental conditions saturation is not observed.

Thus, the hypothesis that ganglion cells, which express Fos in the retina, are afferent to the SCN and linked to the induction of Fos in the SCN seems inappropriate (Servière and Lavialle, 1996). These results agree with a recent study in a rodless transgenic mouse (Lupi et al., 1999) in which constitutive expression of Fos in the retina remains constant, whereas in the $\mathrm{SCN}$, Fos levels vary according to irradiance.

\section{Photic response to total photons in the SCN and the retina}

Figure 3 shows the responses plotted in the SCN as a function of total number of photons in the stimulus. In the SCN, the NakaRushton curve fit shows a proportional increase in the response of light-induced Fos expression to irradiance levels, from $\sim 10^{12}$ to $10^{17}$ photons $/ \mathrm{cm}^{2}$. From $10^{10}$ to $10^{12}$ photons $/ \mathrm{cm}^{2}$, Fos expression is significantly higher than in dark controls (Fig. 4), although no increase with increased irradiance is observed over at least $2 \log$ units. The response saturates above $10^{17}$ photons $/ \mathrm{cm}^{2}$. The photon values obtained from the duration series fall in the range of $10^{12}$. $10^{15}$ photons $/ \mathrm{cm}^{2}$, below that required for saturation, which explains why in Figure $1 B$ the response shows no saturation in our conditions.

The response curve for Fos induction as a function of photon number suggests a reciprocal relationship over $5 \log$ units between the irradiance and duration of the light stimulation from $10^{12}$ to $10^{17}$ photons $/ \mathrm{cm}^{2}$ in the SCN. The slope of the function was 0.44 (similar to the slopes for irradiance and duration above), and the total number of photons necessary to induce a half saturation was $1 \times 10^{15}$ photons $/ \mathrm{cm}^{2}$.

The response was completely different in the retina (curve not shown). A significant Fos induction in the ganglion cell layer was only observed starting at $3.8 \times 10^{14}$ photons $/ \mathrm{cm}^{2}$, and the response saturates rapidly (1 log unit higher). The slope was 1.0 , and the half saturation value was $9.9 \times 10^{14}$ photons $/ \mathrm{cm}^{2}$. The two curves differ in terms of threshold, slope, and saturation value. Compared with the SCN, the response for the retina has a steeper slope and a higher threshold (several log units) but a lower half saturation value.

\section{DISCUSSION}

\section{Irradiance response for Fos induction in the SCN}

The amplitude of Fos induction and of a behavioral phase shift both increase with increased irradiance, although the responses differ in threshold, saturation, and range. These differences may be related to a number of factors, including previous exposure to constant darkness (Shimomura et al., 1998) or other feedback mechanisms. For example, in the tau mutant hamster, the amplitude of a phase shift increases significantly after a long period (49 d) in constant darkness compared with a short period (2 d), whereas the amplitude of the Fos response remains similar regardless of the time spent in the dark (Shimomura et al., 1998). This argues that the magnitude of Fos response to light is more tightly linked to the direct photic input to the circadian clock, whereas the output locomotor response depends on feedback or neural integration at subsequent stages of the clock mechanism. This is consistent with the observation that although the amplitude of a phase shift to different irradiances differs between wild-type and rodless transgenic mice, the amplitude of Fos expression in both strains remains 

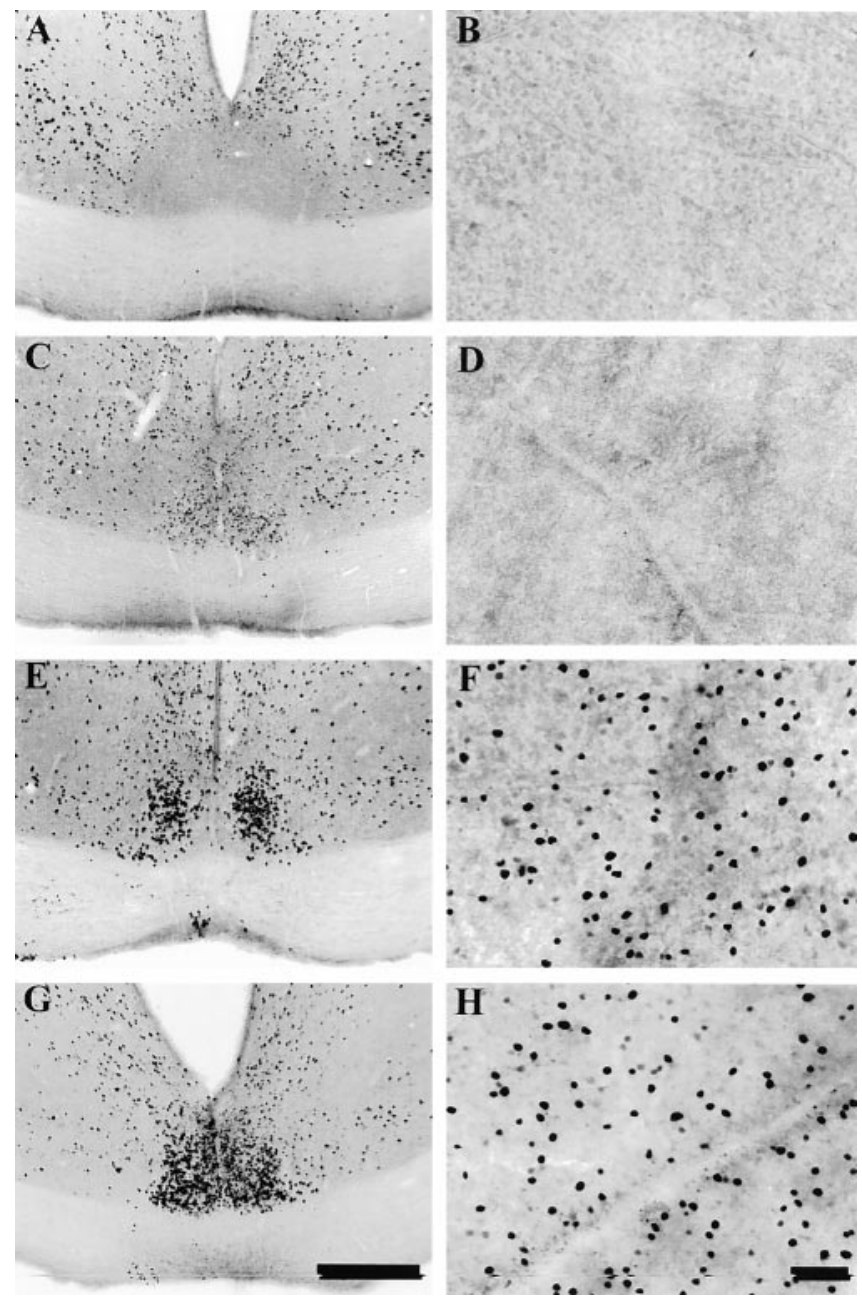

Figure 4. Photomicrographs showing the distribution of Fosimmunoreactive nuclei in the $\operatorname{SCN}(A, C, E, G)$ and in flat-mounted retina from the same animals $(B, D, F, H)$. Animals were placed in the light apparatus at CT16 and either exposed to no light (dark controls, $A$ and $B$ ) or given monochromatic light pulses $(15 \mathrm{~min}, \lambda=500 \mathrm{~nm}$,$) of increasing$ irradiance: $0.95 \times 10^{9}(C, D)$ or $0.95 \times 10^{13}(E, F)$ or $0.95 \times 10^{15}$ photons per $\mathrm{cm}^{2} / \mathrm{sec}(G, H)$. Scale bars: $200 \mu \mathrm{m}$ (SCN); $40 \mu \mathrm{m}$ (retina). At saturation levels the entire SCN is filled with densely stained neurons primarily located in the ventral region of the $\operatorname{SCN}(G)$. In animals that received lower levels of irradiance, the number of Fos-positive cells is diminished and more restricted in distribution $(C, E)$. In control animals, none or only a few lightly stained neurons are seen in the nucleus $(A)$, and the total optical density of the entire nucleus is low. In all cases, Fospositive cells are also observed in the lateral hypothalamic region and in the periventricular region immediately dorsal to the SCN, extending toward the periventricular nuclei.

proportional to photon number (Lupi et al., 1999). Finally, a number of studies show that, for an equivalent level of irradiance, certain anesthetics or phamacological agents can diminish the amplitude of the phase shift without affecting the amplitude of Fos induction (Colwell et al., 1993a,b).

As a consequence, the kinetics of the response characteristics of different outputs of the clock differ in terms of threshold, range, and saturation (Fig. 5). In addition to reflecting distinctions in the inherent mechanisms of each response, these differences may be related to the resolution of the technique used. For example, the 5-10 min sampling intervals used for behavioral phase shifts offer low resolution, whereas quantitative measures of Fos distinguish fine relative levels of expression in individual cells. Furthermore, because Fos reflects cellular activity at the input stage of the clock, it is not unexpected that the range is greater than other outputs at subsequent stages of neural integration.

Figure 6 further illustrates the difference in the significance of

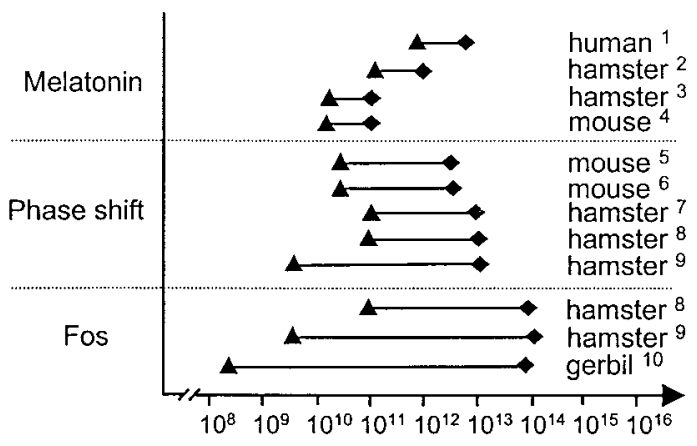

Irradiance (photons $/ \mathrm{cm}^{2} / \mathrm{s}$ )

Figure 5. Comparison of different measures of circadian responses (melatonin suppression, phase shift of locomotor activity, and Fos expression) in relation to irradiance. This figure summarizes results of the present and previous studies of responses of the circadian system. Only those studies using monochromatic light $(500-515 \mathrm{~nm})$ are used to provide valid comparisons in terms of irradiance (photon flux). The range (solid line) and irradiance levels required for both threshold $(\boldsymbol{\Lambda})$ and saturation $(\bullet)$ for the different responses are indicated. In general, threshold levels are lower and the range of the response greater in more recent studies than those obtained in earlier investigations, possibly because of differences in methodological or conceptual approaches. In terms of threshold irradiance required to induce a response, melatonin suppression, a behavioral phase shift, and Fos induction show roughly similar threshold values, between $10^{9}$ and $10^{10}$ photons per $\mathrm{cm}^{2} / \mathrm{sec}$. The threshold in our study is slightly lower $\left(10^{8}\right.$ photons per $\mathrm{cm}^{2} / \mathrm{sec}$ ), possibly because similar low levels of irradiance have not been examined previously. Fos induction shows the widest response range and highest saturation value. In contrast, melatonin suppression has a very narrow range compared with a behavioral shift and Fos induction and rapidly reaches saturation levels at $10^{11}$ photons per $\mathrm{cm}^{2} / \mathrm{sec}$. [1, Brainard et al. (1988); 2, Podolin et al. (1987); 3, Nelson and Takahashi (1991b); 4, Lucas et al. (1999); 5, Foster et al. (1991); 6, Yoshimura and Ebihara (1996); 7, Nelson and Takahashi (1991a); 8, Kornhauser et al. (1990); 9, Zhang et al (1996); 10, present study.]

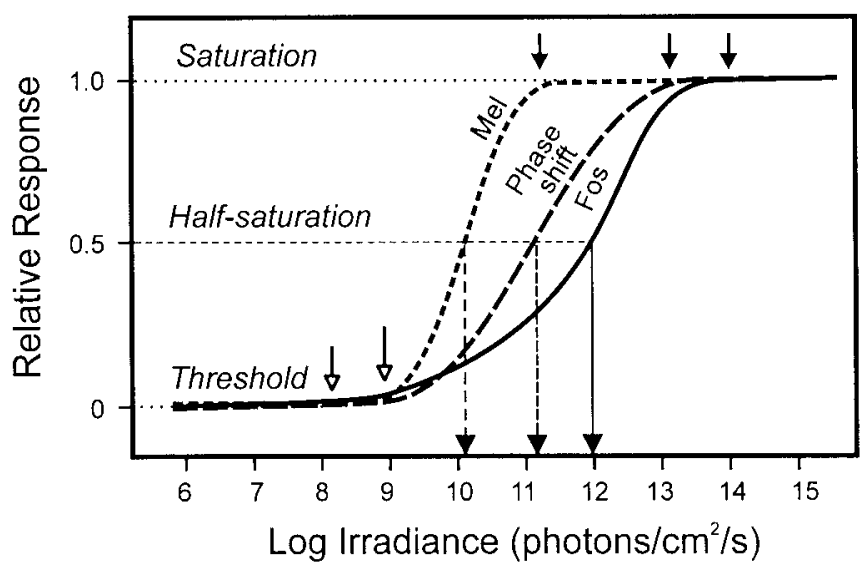

Figure 6. Schematic representation of differences in sensitivity and threshold for melatonin suppression, phase shift of locomotor activity, and Fos expression in relation to irradiance (from Fig. 5). The term "sensitivity" is an aspect of response probability and refers to the reciprocal of the irradiance value required to induce a one-half maximum response (see vertical lines that intersect the half saturation line). The sensitivity value depends on the range (threshold-saturation) and slope of the response. Using this definition, melatonin suppression is the most sensitive response. Threshold refers to the irradiance required to induce a response statistically different from a control value. In terms of threshold (shown as open arrows), Fos induction requires the lowest irradiance. The range of response for Fos induction is greatest because the threshold is low and saturation is high (shown as closed arrows).

response sensitivity and threshold sensitivity. The former term expresses the probability of response to changes in irradiance and has usually been used to express the relative degree of sensitivity for responses of the circadian system. Applied in this sense, the response for melatonin suppression is more sensitive than a phase shift, whereas induction of Fos is the least sensitive. In terms of 
threshold, on the contrary, Fos induction is observed at 1-2 log units lower than melatonin suppression or a behavioral phase shift. This distinction is particularly well illustrated by comparison of Fos induction in the SCN and in the retina. In terms of response sensitivity the retina is more sensitive, but this definition of sensitivity appears paradoxical because at irradiance levels when no response is observed in the retina, the SCN shows robust Fos induction.

\section{Temporal response for Fos induction in the SCN}

The minimal duration required for obtaining a response in the circadian system has not been well studied, and the response differs depending on whether one considers a phase shift or entrainment. Entrainment of locomotor activity to brief stimuli $(0.5-1000 \mathrm{msec})$ has been observed in both nocturnal squirrels and bats (DeCoursey, 1972; Joshi and Chandrashekaran, 1984). In contrast, Nelson and Takahashi (1991a) originally reported that a behavioral phase shift could not be induced using very short duration $(3 \mathrm{msec})$ light flashes. The authors suggested that this lack of responsiveness reflects the inability of the circadian system or some component of the transduction pathway to detect or integrate very short duration pulses of light. Van den Pol et al. (1998) and Arvanitogiannis and Amir (1999) subsequently demonstrated that although a single short (10 $\mu \mathrm{sec}-2 \mathrm{msec})$ bright flash fails to induce a phase shift, trains of pulses produce a phase shift similar to a continuous light stimulus if the total energy falls into the appropriate range. In the latter study only the response for Fos remains proportional to total stimulus duration, indicating integration of photons. The lack of response to a single short pulse is also coherent with the fact that some SCN neurons that initially show no electrophysiological response to a single light stimulation can respond to both repeated flashes and to continuous 1-2 min light durations (Meijer et al., 1986).

\section{Photon integration and reciprocity in the circadian system}

The idea of reciprocity in the circadian system, first suggested by Takahashi et al. (1984), implies that the magnitude of the phase shift depends on the total number of photons received by the retina independent of irradiance or duration. This concept is of fundamental importance because it implies the existence of a cellular mechanism capable of integrating, or counting, photons over long durations without becoming nonlinear. For a given stimulus duration, the amplitude of a phase shift was proportional to the number of photons in the light stimulus, but for durations $<30 \mathrm{sec}$ or $>300$ sec, sensitivity was reduced. Furthermore, at equivalent irradiances the amplitude of the phase shift to $1 \mathrm{hr}$ pulses was usually larger than for $300 \mathrm{sec}$ pulses, whereas when considered as a function of equivalent numbers of photons, the amplitudes of the response at 1 hr were less than at $300 \mathrm{sec}$. As a result the authors concluded that "the hamster circadian system appears unable to integrate light information in pulses longer than $300 \mathrm{sec}$." Nelson and Takahashi (1991a) suggest that this reduced sensitivity occurs downstream from the retina and neural pathway to the SCN, because electrophysiological studies (Meijer et al., 1986) show that SCN neurons can maintain response levels over sustained periods of time, with little or no loss of sensitivity. However, a more recent study shows that the circadian system can integrate the number of photons in single or multiple pulses of light within a $1 \mathrm{hr}$ time interval (Nelson and Takahashi, 1999).

We demonstrate that the response of the circadian system for Fos induction presents a reciprocal relationship for the total number of photons within a range of $3 \mathrm{sec}$ to $47.5 \mathrm{~min}$. The amplitude of the response can be predicted from a single linear function, based on photon number. This relation of reciprocity holds for 5 $\log$ units of photon number and is reported for the first time at a cellular level. This broad range of temporal integration and reciprocity is a unique feature of the circadian system and is coherent with, and predictable from, the known luminance response properties of SCN neurons (Meijer et al., 1986). Individual neurons show a monotonic increase in discharge rate as a function of irradiance and sustained activation for periods exceeding $1 \mathrm{hr}$. Many neurons fail to attain saturation even at the highest stimulus values (800 lux, white light). We speculate that the difference between the linear temporal integration of photons for Fos induction, and the duration dependent sensitivity for a phase shift, may be related to feedback mechanisms at subsequent stages of neural treatment.

The site of integration might occur at one or several possible steps in the transduction pathway from the photoreceptors to the clock. The data from the present study support the idea that the site of integration occurs within the SCN rather than in the retina. However, the hypothesis that some photic integration may occur in retina cannot be excluded. The retinal ganglion cells that project to the SCN may express a different early gene or have a different response threshold. For example, the retinohypothalamic ganglion cells may have a higher threshold (or different time course) for Fos induction than early gene expression in the SCN. In addition, we use monochromatic green light $(500 \mathrm{~nm})$, and the response may be different for other wavelengths, in particular if opponent photoreceptor mechanisms are involved upstream. Furthermore, certain aspects of retinal photostasis (rhodopsin levels, rod outer segment length) imply the existence of long-term integrative physiological mechanisms, which may ultimately be linked to circadian photoreception (Schremser and Williams, 1995). Because reciprocity is observed in the expression of an immediate early gene, the protooncogene $c$-fos may either reflect some aspect of the cellular mechanism of photic integration or be an essential factor in the mechanism of integration. It is unknown whether certain clock gene homologs for which expression depends on circadian phase and irradiance (similar to Fos) may also play a role in photic integration. For example, mammalian homologs of the Drosophila clock gene period (per) have recently been characterized in several studies (Albrecht et al., 1997; Shearman et al., 1997; Shigeyoshi et al., 1997; Tei et al., 1997). Moreover, mPer1 and mPer2 RNA levels exhibit circadian rhythms in the SCN (Albrecht et al., 1997; Shearman et al., 1997; Tei et al., 1997), and both are induced by light exposure during subjective night but not during subjective day (Albrecht et al., 1997; Shearman et al., 1997; Shigeyoshi et al., 1997). mPer3, a clock-relevant member of the mammalian Per gene family, has been cloned and characterized more recently (Zylka et al., 1998), but unlike mPer1 and mPer2, mPer3 RNA levels are not induced by light pulses during subjective night. Because mPerl mRNA increases with increasing irradiance and the degree of induction is correlated with the relative amount of phase shift of locomotor activity (Shigeyoshi et al., 1997), this clock gene could putatively participate in the mechanism of photon integration. In addition, because the kinetics of $c$-fos and mPerl appear to be more or less simultaneous, it is possible that light-induced Fos may have synergistic effects in accelerating the transcription of mPerl. However, the potential role of Fos in the induction of $m P e r$ awaits clarification (Best et al., 1999).

The present results also have important implications related to the nature of the irradiance detection system. The capacity for an extensive range of temporal integration by the circadian system is unusual compared with responses of the visual or other sensory systems (Nelson and Takahashi, 1999). Temporal summation in the visual system is limited to a few hundred milliseconds (Baumgardt, 1972). A $5 \log$ unit capacity for stimulus integration is unique for biological systems, with the possible exception of the effect of light on plant germination, involving phytochromes (Cone et al., 1985). The capacity for temporal integration must be compatible with the photopigment properties and/or the organization of the neural input pathway to the SCN. Previous studies have considered that a cone-like photoreceptor could be involved in circadian photoreception, based on the threshold and range of cone sensitivity (Nelson and Takahashi, 1991a) and on the conservation of circadian responses in rodless $r d$ (Provencio et al., 1994, 1998) and transgenic (Lupi et al., 1999) mice. In comparison, the threshold for human cones is $10^{9}$ photons per $\mathrm{cm}^{2} / \mathrm{sec}$, and a steady state of cone 
saturation occurs between $10^{13}$ and $10^{15}$ photons per $\mathrm{cm}^{2} / \mathrm{sec}$, values similar to the response range of the circadian system. However, more recent evidence from rodless-coneless transgenic mice suggests that a novel non-rod, non-cone photopigment may be involved in circadian photoreception (Freedman et al., 1999; Lucas et al., 1999). Putative photopigments include homologs of VA opsin (Soni et al., 1998), melanopsin (Provencio et al., 1998), or the cryptochromes (Miyamoto and Sancar, 1998), which may show different phototransduction properties (Soni and Foster, 1997). Definition of the response properties of the photopigment and of the functional architecture of the neural network involved in irradiance detection are necessary to determine the mechanisms of photon integration.

\section{REFERENCES}

Albrecht U, Sun Z, Eichele G, Lee C (1997) A differential response of two putative mammalian circadian regulators, mper1 and mper 2 , to light. Cell 91:1055-1064.

Aronin N, Sagar SM, Sharp FR, Schwartz WJ (1990) Light regulates expression of a fos-related protein in rat suprachiasmatic nuclei. Proc Natl Acad Sci USA 87:5959-5962.

Arvanitogiannis A, Amir S (1999) Resetting the rat circadian clock by ultra-short light flashes. Neurosci Lett 261:159-162.

Bauer MS (1992) Irradiance responsivity and unequivocal type- 1 phase responsivity of rat circadian activity rhythms. Am J Physiol 263:R1110-R1114.

Baumgardt E (1972) Threshold quantal problems. In: Handbook of sensory physiology, visual psychophysics, Vol V II (Jameson D, Hurvich LM, eds), pp 29-55. Berlin: Springer.

Best JD, Maywood ES, Smith KL, Hastings MH (1999) Rapid resetting of the mammalian circadian clock. J Neurosci 19:828-835.

Boivin D, Duffy J, Kronauer R, Czeisler C (1996) Dose-response relationships for resetting of human circadian clock by light. Nature 379:540-542.

Brainard GC, Lewy AJ, Menaker M, Fredrickson RH, Miller LS, Weleber RG, Cassone V, Hudson D (1988) Dose-response relationship between irradiance and the suppression of plasma melatonin in human volunteers. Brain Res 454:212-218.

Chambille I, Doyle S, Servière J (1993) Photic induction and circadian expression of fos-like protein. Immunohistochemical study in the retina and suprachiasmatic nuclei of hamster. Brain Res 612:138-150.

Colwell CS, Foster RG (1992) Photic regulation of fos-like immunoreactivity in the suprachiasmatic nucleus of the mouse. J Comp Neurol 324:135-142.

Colwell CS, Kaufman CM, Menaker M (1993a) Photic induction of Fos in the hamster suprachiasmatic nucleus is inhibited by baclofen but not by diazepam or bicucullin. Neurosci Lett 163:177-181.

Colwell CS, Kaufman CM, Menaker M, Ralph MR (1993b) Lightinduced phase shifts and Fos expression in the hamster circadian system: the effects of anesthetics. J Biol Rhythms 8:179-188.

Cone JW, Jaspers PAPM, Kendrick RE (1985) Biphasic fluence-response curves for light-induced germination of Arabidopsis thaliana seeds. Plant Cell Environ 8:605-612.

Cooper HM, Dkhissi O, Sicard B, Groscarret H (1998) Light-evoked c-fos expression in the SCN is different under on/off and twilight conditions. In: Biological clocks: mechanisms and applications (Touitou Y, ed), pp 181-188. Amsterdam: Elsevier Science.

DeCoursey PJ (1972) LD ratios and the entrainment of a circadian activity in a nocturnal and a diurnal rodent. J Comp Physiol 78:221-235.

Dodt E, Echte K (1961) Dark and light adaptation in pigmented and white rat as measured by electroretinogram threshold. J Neurophysiol 24:427-445.

Earnest DJ, Iadarola M, Yeh HH, Olschowka JA (1990) Photic regulation of $c$-fos expression in neural components governing the entrainment of circadian rhythms. Exp Neurobiol 109:353-361.

Emerson VF (1980) Grating acuity in the golden hamster: the effects of stimulus orientation and luminance. Exp Brain Res 38:43-52.

Foster R, Provencio I, Hudson D, Fiske S, DeGrip W, Menaker M (1991) Circadian photoreception in the retinally degenerate mouse $(r d / r d)$. J Comp Physiol [A] 169:39-50.

Freedman MS, Lucas RJ, Soni B, von Schantz M, Munoz M, David-Gray Z, Foster R (1999) Regulation of mammalian circadian behavior by nonrod, non-cone, ocular photoreceptors. Science 284:502-504.

Jojich L, Pourcho RG (1996) Glutamate immunoreactivity in the cat retina: a quantitative study. Vis Neurosci 13:117-133.

Joshi D, Chandrashekaran MK (1984) Bright light flashes of 0.5 milliseconds reset the circadian clock of a microchiropteran bat. J Comp Exp Zool 230:325-328.

Kornhauser JM, Nelson DE, Mayo KE, Takahashi JS (1990) Photic and circadian regulation of $c$-fos gene expression in the hamster suprachiasmatic nucleus. Neuron 5:127-134.

Kornhauser JM, Nelson DE, Mayo KE, Takahashi JS (1992) Regulation of jun- $B$ messenger RNA and AP-1 activity by light and a circadian clock. Science 255:1581-1584.

Le Grand Y (1968) Light, colour and vision (Hunt RWG, Walsh JWT, Hunt FRW, eds). London: Chapman and Hall.

Lucas RJ, Freedman MS, Munoz M, Garcia-Fernandez JM, Foster RG (1999) Regulation of the mammalian pineal by non-rod, non-cone, ocular photoreceptors. Science 284:505-507.

Lupi D, Cooper H, Froechlich A, Standford L, McCall M, Foster R (1999) Transgenic ablation of rod photoreceptors alters the circadian phenotype of mice. Neuroscience 89:363-374.

Meijer JH, Groos GA, Rusak B (1986) Luminance coding in a circadian pacemaker: the suprachiasmatic nucleus of the rat and hamster. Brain Res 382:109-118.

Miyamoto Y, Sancar A (1998) Vitamin B2-based blue-light photoreceptors in the retinohypothalamic tract as the photoactive pigments for setting the circadian clock in mammals. Proc Natl Acad Sci USA 95:6097-6102.

Nabors LB, Songu-Mize E, Mize RR (1988) Quantitative immunohistochemistry using an image analyzer. II. Concentration standards for transmitter immunohistochemistry. J Neurosci Methods 26:25-34.

Naka KI, Rushton WAH (1966) S-potentials from luminosity units in the retina of fish (Cyprinidae). J Physiol (Lond) 185:587-599.

Nelson DE, Takahashi JS (1991a) Sensitivity in a visual pathway for circadian entrainment in the hamster (Mesocricetus auratus). J Physiol (Lond) 439:115-145.

Nelson DE, Takahashi JS (1991b) Comparison of visual sensitivity for suppression of pineal melatonin and circadian phase-shifting in the golden hamster. Brain Res 554:272-277.

Nelson DE, Takahashi JS (1999) Integration and saturation within the circadian photic entrainment pathway of hamsters. Am J Physiol 46:R1351-R1361.

Nir I, Agarwal N (1993) Diurnal expression of c-fos in the mouse retina. Mol Brain Res 19:47-54.

Pittendrigh CS (1981) Circadian systems: entrainment. In: Biological rhythms. Handbook of behavioral neurobiology (Aschoff J, ed), pp 95124. New York: Plenum.

Podolin PL, Rollag MD, Brainard GC (1987) The suppression of nocturnal pineal melatonin in the syrian hamster: dose-response curves at 500 and $360 \mathrm{~nm}$. Endocrinology 121:266-270.

Provencio I, Wong S, Lederman AB, Argamaso SM, Foster RG (1994) Visual and circadian responses to light in aged retinally degenerate mice. Vision Res 34:1799-1806.

Provencio I, Jiang G, De Grip WJ, Par Hayes W, Rollag MD (1998) Melanopsin: an opsin in melanophores, brain and eye. Proc Natl Acad Sci USA 95:340-345.

Rea MA, Buckley B, Lutton LM (1993) Local administration of EAA antagonists blocks light-induced phase shifts and c-fos expression in hamster SCN. Am J Physiol 265:R1191-R1198.

Rusak B, Robertson HA, Wisden W, Hunt SP (1990) Light pulses that shift rhythms induce gene expression in the suprachiasmatic nucleus. Science 248:1237-1240.

Rusak B, McNaughton L, Robertson HA, Hunt SP (1992) Circadian variation in photic regulation of immediate-early gene mRNAs in rat suprachiasmatic nucleus cells. Brain Res Mol Brain Res 14:124-130.

Sagar SM, Sharp FR (1990) Light induces a Fos-like nuclear antigen in retinal neurons. Brain Res Mol Brain Res 7:17-21.

Schremser J-L, Williams TP (1995) Rod outer segment (ROS) renewal as a mechanism for adaptation to a new intensity environment. I. Rhodopsin levels and ROS length. Exp Eye Res 61:17-24.

Schwartz WJ, Takeuchi J, Shannon W, Davis EM, Aronin N (1994) Temporal regulation of light-induced fos and fos-like protein expression in the ventrolateral subdivision of the rat suprachiasmatic nucleus. Neuroscience 58:573-583.

Servière S, Lavialle M (1996) Le noyau suprachiasmatique: approche cellulaire du fonctionnement de l'horloge. Pathol Biol 44:497-508.

Shearman LP, Zylka MJ, Weaver DR, Kolakowski Jr LF, Reppert SM (1997) Two period homologs: circadian expression and photic regulation in the suprachiasmatic nuclei. Neuron 19:1261-1269.

Shigeyoshi Y, Taguchi K, Yamamoto S, Takekida S, Yan L, Tei H, Moriya T, Shibata S, Loros J, Dunlap J, Okamura H (1997) Light-induced resetting of a mammalian circadian clock is associated with rapid induction of mPer1 transcript. Cell 91:1043-1053.

Shimomura K, Kornhauser JM, Wisor JP, Umezu T, Yamazaki S, Ihara NL, Takahashi JS, Menaker M (1998) Circadian behavior and plasticity of light-induced c-fos expression in SCN of tau mutant hamsters. J Biol Rhythms 13:305-314.

Sicard B, Diarra W, Cooper HM (1999) Ecophysiology and chronobiology applied to rodent pest management in semiarid agriculture areas in sub-saharan west Africa. In: Ecology based management of rodent pests (Singleton GR, Hinds LA, Liers H, Zhang Z, eds), pp 409-440. Canberra: ACIAR monograph. 
Soni BG, Foster RG (1997) A novel and ancient vertebrate opsin. FEBS Lett 406:279-283.

Soni B, Philip A, Foster R (1998) Novel retinal photoreceptors. Nature 394:27.

Takahashi JS, DeCoursey PJ, Bauman L, Menaker M (1984) Spectra sensitivity of a novel photoreceptive system mediating entrainment of mammalian circadian rhythms. Nature 308:186-188.

Tei H, Okamura H, Shigeyoshi Y, Fukuhara C, Ozawa R, Hirose M, Sakaki Y (1997) Circadian oscillation of a mammalian homologue of the Drosophila period gene. Nature 389:512-516.

Van den Pol A, Cao V, Heller H (1998) Circadian system of mice integrates brief light stimuli. Am J Physiol 44:R654-R657.

Vindlacheruvu RR, Ebling FJP, Maywood ES, Hastings MH (1992) Blockade of glutamatergic neurotransmission in the suprachiasmatic nucleus prevents cellular and behavioral responses of the circadian system to light. Eur J Neurosci 4:673-679.

Wollnik F, Brysch W, Uhlmann E, Gillardon F, Bravo R, Zimmermann M, Schingensiepen KH, Herdegen T (1995) Block of $c$-fos and JunB expres- sion by antisense oligonucleotides inhibits light-induced phase shifts of the mammalian circadian clock. Eur J Neurosci 7:388-393.

Yoshida K, Kawamura K, Imaki J (1993) Differential expression of c-fos mRNA in rat retinal cell: regulation by light/dark cycle. Neuron 10:1049-1054.

Yoshimura T, Ebihara S (1996) Spectral sensitivity of photoreceptors mediating phase-shifts of circadian rhythms in retinally degenerate CBA/J $(r d / r d)$ and normal CBA/N (+/+) mice. J Comp Physiol [A] 178:797-802.

Zhang Y, Kornhauser J, Zee P, Mayo K, Takahashi J, Turek F (1996) Effects of aging on light-induced phase-shifting of circadian behavioral rhythms, Fos expression and CREB phosphorylation in the hamster suprachiasmatic nucleus. Neuroscience 70:951-961.

Zylka MJ, Shearman LP, Weaver DR, Reppert SM (1998) Three period homologs in mammals: differential light responses in the suprachiasmatic circadian clock and oscillating transcripts outside of brain. Neuron 20: 1103-1110. 